\title{
Surface display of p75, a Lactobacillus rhamnosus GG derived protein, on Bacillus subtilis spores and its antibacterial activity against Listeria monocytogenes
}

\author{
Soo Ji Kang, Ji Su Jun, Jeong A Moon and Kwang Won Hong ${ }^{*}$ (D)
}

\begin{abstract}
Lactobacillus rhamnosus p75 protein with peptidoglycan hydrolase (PGH) activity is one of the key molecules exhibiting anti-apoptotic and cell-protective activity for human intestinal epithelial cells. In this study, with the goal of developing new probiotics, the p75 protein was displayed on the surface of Bacillus subtilis spores using spore coat protein CotG as an anchoring motif. The PGH activity, stability, and the antibacterial activity of the spore-displayed p75 (CotG-p75) protein were also investigated. The PGH activity of the CotG-p75 against peptidoglycan extracted from B. subtilis was confirmed by the ninhydrin test. Under various harsh conditions, compared to the control groups, the PGH activities of CotG-p75 were very stable in the range of $\mathrm{pH} 3-7$ and maintained at $70 \%$ at $50^{\circ} \mathrm{C}$. In addition, the antibacterial activity of CotG-p75 against Listeria monocytogenes was evaluated by a time-kill assay. After $6 \mathrm{~h}$ incubation in phosphate-buffered saline, CotG-p75 reduced the number of viable cells of L. monocytogenes by up to 2.0 log. Scanning electron microscopy analysis showed that the cell wall of $L$. monocytogenes was partially damaged by the treatment with CotG-p75. Our preliminary results show that CotG-p75 could be a good candidate for further research to develop new genetically engineered probiotics.
\end{abstract}

Keywords: Spore surface display, p75 protein, Antibacterial activity, L. monocytogenes

\section{Introduction}

Lactobacillus rhamnosus GG (LGG) is one of the most widely studied probiotic strains and has been reported to be effective in treating and preventing ulcerative colitis, diarrhea, and atopic dermatitis in numerous clinical studies (Zocco et al. 2006; Szajewska et al. 2011; Doron et al. 2005). p75, also known as major secreted protein 1 , is one of the soluble proteins derived from LGG and is a key molecule that shows probiotic features. p75 regulates the proliferation and survival of intestinal epithelial cells through stimulation of Akt activation, inhibition of

\footnotetext{
*Correspondence: hkwon@dongguk.edu

Department of Food Science and Biotechnology, College of Life Science and Biotechnology, Dongguk University, Goyang-si 10326, Republic of Korea
}

cytokine-induced epithelial cell apoptosis, promotion of cell growth, and reduction of tumor necrosis factor (TNF)-induced colon epithelial damage (Yan and Polk 2002; Yan et al. 2007). In addition, p75 protects the intestinal epithelial tight junctions and barrier functions from disruption by hydrogen peroxide (Seth et al. 2008). On the other hand, many studies have shown that p75 has sequence homology with peptidoglycan hydrolase (PGH) and that has D-glutamyl-L-lysyl endopeptidase activity (Bäuerl et al. 2010; Claes et al. 2012). However, it is not yet known whether the PGH activity of p75 is directly related to its various probiotic properties mentioned above.

Although probiotics have numerous health benefits including treating and preventing intestinal disease, they have limitations. Because probiotic action is non-specific 
and non-discriminatory, it is affected by strains or dose, and the effect may vary from person to person (Bomba et al. 2002; Karimi and Peña 2008; Morrow and Kollef 2008). Since probiotics are living microorganisms, they tend to have low viability during food processing and passage through the gastrointestinal tract (MattilaSandholm et al. 2002). To overcome these limitations, there have been many efforts to develop new probiotics through bioengineering. To improve stress tolerance, the betaine transporter gene (betL) of $L$. monocytogenes and the trehalose synthesis gene (ostAB) of Escherichia coli were cloned into probiotic strains of Lactobacillus and Bifidobacterium (Sheehan et al. 2006; Termont et al. 2006; Sheehan et al. 2007). In addition, probiotic strains and E. coli were engineered to produce antimicrobial peptides or compounds to enhance its antimicrobial actions (Goh et al. 2012; Chen et al. 2010).

Spore surface display technology has been proposed as an alternative approach to develop new probiotics with improved stability and efficiency. Spore surface display is a technique for displaying foreign proteins on the endospores of spore-forming bacteria. B. subtilis, a non-pathogenic spore-forming bacteria, has been widely used for spore surface displays due to its GRAS (general recognized as safe) status and probiotic features (Elshaghabee et al. 2017; Cutting 2011; Hong et al. 2005). B. subtilis spores can survive in extreme environments due to its unique structure. It is encased in a thick peptidoglycan layer and many coat proteins (Henriques and Moran 2007; Nicholson et al. 2000). With these properties, spore displayed proteins are more stable than free ones (Zhang et al. 2019; Chen et al. 2017; Kim and Schumann 2009). Therefore, spore surface display has been applied in various fields such as vaccine and drug development, wholecell biocatalyst, and multimeric protein production (Zhang et al. 2019). Recently, it has been actively applied in the food industries for production of materials such as D-neuraminic acid (Xu et al. 2011), D-tagatose (Guo et al. 2018; Liu et al. 2014) D-allulose (He et al. 2016), lactulose (Wang et al. 2016), and trehalose (Liu et al. 2019). However, it has not been previously reported that spore surface display technology has been applied to the development of new probiotics.

In this study, we displayed the p75 protein on the surface of $B$. subtilis spores using $\operatorname{Cot} G$ as an anchoring motif to develop a novel probiotic. To confirm the successful expression of the displayed p75 (CotG-p75), the PGH activity of CotG-p75 against peptidoglycan was examined. In addition, to investigate the stability of CotG-p75 on the spore surface, the PGH activity of CotG-p75 was measured under various temperature and $\mathrm{pH}$ conditions. Finally, the antibacterial effect of CotGp75 on Gram positive bacteria was investigated for $L$. monocytogenes, a major food poisoning bacterium.

\section{Materials and methods}

Bacterial strains, growth conditions, and transformation

The bacterial strains used in this study are listed in Table 1. E. coli DH5 $\alpha$ was used for the subcloning experiment and transformation. B. subtilis 168 was used as the

Table 1 Bacterial strains, plasmids, and primers used in this study

\begin{tabular}{|c|c|c|}
\hline Bacterial strains or plasmids & Description & Reference \\
\hline \multicolumn{3}{|l|}{ Bacterial strains } \\
\hline Escherichia coli DH5a & $\begin{array}{l}F-, \varphi \text { 80dlacZMM15, } \triangle(\text { lacZYA-argF)U169, deoR, recA1, endA1, hsdR17(rK-, mK+), phoA, supE44, } \\
\lambda-\text {, thi-1, gyrA96, relA1 }\end{array}$ & Hanahan (1983) \\
\hline Bacillus subtilis 168 & $\operatorname{trp} C 2$ & $\begin{array}{l}\text { Anagnostopoulos } \\
\text { and Crawford } \\
\text { (1961) }\end{array}$ \\
\hline Lactobacillus rhamnosus GG & ATCC 53103 & Purchased from ATCC \\
\hline Listeria monocytogenes & ATCC19115 & Purchased from ATCC \\
\hline \multicolumn{3}{|l|}{ Plasmids } \\
\hline pUB19 & E. coli-B. subtilis shuttle vector, $\mathrm{Ap}^{\mathrm{r}}, \mathrm{Km}^{\mathrm{r}}$ & Kang et al. (2019) \\
\hline pUB19-cotG-p75 & Spore display of p75 using the CotG anchor & This study \\
\hline \multicolumn{3}{|l|}{ Primers } \\
\hline CotG-F & 5'-CTTCGACGCGTCAGCTGGCACC-3' & This study \\
\hline CotG-R & 5'-GTCCCTGTCGAGCTTCCTCCTCCTCCTTTGTATTTCTTTTTGGACTACCCAGCAATTGCCGT-3' & This study \\
\hline P75-F & 5'-AGAAATACAAAGGAGGAGGAGGAAGCTCGACAGGGACGGTCAGTTACAAATCCGG-3' & This study \\
\hline P75-R & 5'-AAGGAAAAAGCGGCCGCTTATAGTGACGGGCGAACCGCAAAGTCAGG-3' & This study \\
\hline
\end{tabular}


host strain to display the p75 protein on its endospore surface. E. coli and B. subtilis were routinely grown in Luria-broth (LB) medium at $37{ }^{\circ} \mathrm{C}$ in a shaking incubator. L. rhamnosus GG ATCC 53103 was used to obtain the p75 gene and it was cultured in Man-Rogosa-Sharpe (MRS) medium at $37{ }^{\circ} \mathrm{C}$. Listeria monocytogenes ATCC 19115 was cultured in Brain Heart Infusion (BHI) broth at $37{ }^{\circ} \mathrm{C}$ in a shaking incubator. In addition, polymyxin acriflavine lithium chloride ceftazidime aesculin mannitol agar was used as a selective medium for L. monocytogenes in the time-kill assay. The transformation of E. coli $\mathrm{DH} 5 \alpha$ used the $\mathrm{CaCl}_{2}$-mediated transformation method described elsewhere (Sambrook et al. 1989). The recombinant plasmid was transformed into $B$. subtilis 168 by the method of Juhas and Ajioka (2016). Antibiotics, ampicillin $(50 \mu \mathrm{g} / \mathrm{mL})$ and kanamycin $(10 \mu \mathrm{g} / \mathrm{mL})$, were added into the medium for the selection of the $E$. coli and B. subtilis cells harboring the pUB19-cotG-p75, respectively.

\section{Plasmid construction}

The primers and plasmid used in this study are listed in Table 1 . The $\cot G$ gene, containing its promoter region and the protein coding region, was amplified by polymerase chain reaction (PCR) using the B. subtilis 168 chromosome as a template and primer sets of $\cot$ G-F/cotG-R. The DNA fragment containing the $p 75$ structural gene except 96 bp of the signal peptide sequence was amplified with primer sets p75-F/p75-R using the Lactobacillus rhamnosus GG (LGG) chromosome as a template. Fragments of the amplified $\cot G$ and $p 75$ genes were fused by overlap extension PCR with oligonucleotide pair cotG-F/p75-R. A flexible linker (Gly-Gly-Gly-Gly-Ser) was inserted between the C-terminus of the CotG protein and the $\mathrm{N}$-terminus of the $\mathrm{p} 75$ protein to improve the structural flexibility of the fused protein (Wriggers et al. 2005). The amplified cotG-p75 DNA (2264 bp) was digested with $M l u \mathrm{I}$ and NotI restriction enzymes and ligated into the shuttle vector pUB19 $\left(\mathrm{Km}^{\mathrm{r}}\right)$ digested with the same enzymes. The resulting plasmid was named pUB19-cotG-p75.

\section{Preparation of spores}

The recombinant B. subtilis 168 containing the pUB19$\cot G-p 75$ was cultured for $62 \mathrm{~h}$ at $37^{\circ} \mathrm{C}$ in Difco Sporulation Medium (DSM) in an incubator shaking at $150 \mathrm{rpm}$. Spores were harvested and prepared as described previously (Nicholson and Setlow 1990). The spores were resuspended in $50 \mathrm{mM}$ sodium phosphate buffer $(\mathrm{pH}$ 7.2) and treated with lysozyme at a final concentration of $0.05 \%$ for $1 \mathrm{~h}$ at $4{ }^{\circ} \mathrm{C}$ to destroy any residual vegetative cells. Then the spores were washed 5 times with $50 \mathrm{mM}$ sodium phosphate buffer to remove any residual lysozyme. The colony forming units (CFU) of the spores were measured by plating serial dilutions on LB agar medium containing kanamycin $(10 \mu \mathrm{g} / \mathrm{mL})$. The final concentration of spores in the buffer was adjusted to $10^{9} \mathrm{CFU} / \mathrm{mL}$.

\section{Preparation of peptidoglycan}

Peptidoglycan (PG) was prepared from the mid-log phase of B. subtilis as described previously by Atrih et al. (1999) with a slight modification. Bacterial cell cultures $(250 \mathrm{~mL})$ were boiled and then centrifuged at $14,000 \times g$ at $4{ }^{\circ} \mathrm{C}$ for $8 \mathrm{~min}$. The pellet was resuspended in $5 \%$ (W/V) hot sodium dodecyl sulfate (SDS) and boiled for $25 \mathrm{~min}$. After centrifugation at the same condition, the pellet was resuspended in $4 \%(\mathrm{~W} / \mathrm{V})$ SDS and boiled for $15 \mathrm{~min}$. The insoluble material was collected by centrifugation and washed with distilled water (DW) more than six times to eliminate all of the SDS. Proteinase K (2 mg/ $\mathrm{mL})$ and trypsin $(200 \mu \mathrm{g} / \mathrm{mL})$ were added for $1 \mathrm{~h}$ and $16 \mathrm{~h}$, separately, at $37^{\circ} \mathrm{C}$ to remove covalently attached proteins. Then the insoluble material was recovered by centrifuge and treated in $48 \%(\mathrm{~V} / \mathrm{V})$ hydrofluoric acid for $24 \mathrm{~h}$ at $4{ }^{\circ} \mathrm{C}$. After recovery by centrifugation, the insoluble cell wall was resuspended in $50 \mathrm{mM}$ Tris- $\mathrm{HCl}$ buffer ( $\mathrm{pH} 7$ ) and washed with cold DW at least five times to adjust it to a neutral $\mathrm{pH}$. Then, the PG extract was suspended in $1.5 \mathrm{~mL} \mathrm{DW}$ and stored at $4{ }^{\circ} \mathrm{C}$.

\section{PGH activity assay of CotG-p75}

Identification of the PGH activity of CotG-p75 was carried out using the ninhydrin method and the B. subtilis PG extract as the substrate (Moore and Stein 1948). In brief, $30 \mu \mathrm{L}$ of CotG-p75 at different concentrations $\left(1.2,1.8,2.4,3.0\right.$, and $\left.3.6 \times 10^{5} \mathrm{CFU} / \mathrm{mL}\right)$ was mixed with $30 \mu \mathrm{L}$ of PG extract. After $15 \mathrm{~min}$ incubation at $37^{\circ} \mathrm{C}$, the mixtures were centrifuged at 13,000 rpm for $5 \mathrm{~min}$ to precipitate any unhydrolyzed PG extract. Then, $30 \mu \mathrm{L}$ of the supernatant was placed in a new tube and reacted with $3 \mu \mathrm{L}$ of $2 \%(\mathrm{~W} / \mathrm{V})$ ninhydrin solution. The reaction solution was heated for $5 \mathrm{~min}$ at $100{ }^{\circ} \mathrm{C}$ and then cooled to room temperature. The absorbance of the reaction mixture was measured at $570 \mathrm{~nm}$ with a Nanodrop 2000 spectrophotometer (Thermo Fisher Scientific, Wilmington, DE, USA). PG treated with DW instead of CotG-p75 was used as a blank. CotG-p75 reacted with DW instead of PG extract was used as the control. The enzymatic activity was measured three times with independent samples.

\section{Stability test}

The stability of CotG-p75 was determined under different temperature and $\mathrm{pH}$ conditions by the ninhydrin method. To identify its thermal stability, $30 \mu \mathrm{L}$ of spores 
$\left(3.6 \times 10^{5} \mathrm{CFU} / \mathrm{mL}\right)$ were heated at each temperature $\left(40,50,60,70\right.$, and $\left.80{ }^{\circ} \mathrm{C}\right)$ for $15 \mathrm{~min}$ using a heating block. Then, all samples were cooled to room temperature $\left(25^{\circ} \mathrm{C}\right)$. To evaluate its $\mathrm{pH}$ stability, $30 \mu \mathrm{L}$ of spores were treated with Mcilvaine's buffer $(\mathrm{pH} 2,3,4,5,6,7$, and 8) for $15 \mathrm{~min}$. Then all samples were washed three times with phosphate-buffered saline (PBS, $\mathrm{pH}$ 7.4). After each treatment, the sample was incubated with the PG extract at $37{ }^{\circ} \mathrm{C}$ for $15 \mathrm{~min}$. Then, the PGH activity of each sample was measured with the ninhydrin method as described above. The relative PGH activity was calculated by defining the activity measured at $25{ }^{\circ} \mathrm{C}$ and $\mathrm{pH} 7$ as $100 \%$.

\section{Antibacterial evaluation}

The antimicrobial effect of CotG-p75 was identified using a time-kill assay based on the National Committee for Clinical Laboratory Standards guidelines (Barry et al. 1999). To identify the antimicrobial effect of CotG-p75 against pathogenic Gram-positive bacteria, L. monocytogenes ATCC 19115 was used. L. monocytogenes was grown to the log phase in $\mathrm{BHI}$ broth at $37^{\circ} \mathrm{C}$. The cells were harvested and washed three times with PBS. The cell pellets were resuspended and adjusted to $10^{8} \mathrm{CFU} /$ $\mathrm{mL}$ with PBS. The time-kill assay was performed in PBS where spores could not germinate and the pathogen could not grow during the incubation period.

To test the antibacterial activity of CotG-p75 against L. monocytogenes, the ratio of CotG-p75 to L. monocytogenes was adjusted to $0.1,1$, and 10 . One $\mathrm{mL}$ each of the L. monocytogenes $\left(10^{8} \mathrm{CFU} / \mathrm{mL}\right)$ and CotG-p $75\left(10^{7}, 10^{8}\right.$, and $10^{9} \mathrm{CFU} / \mathrm{mL}$ ) were mixed together in a $5 \mathrm{~mL}$ Eppendorf tube and incubated at $37^{\circ} \mathrm{C}$ for up to $6 \mathrm{~h}$. Wildtype spores and PBS with no spores were mixed with $10^{8} \mathrm{CFU} / \mathrm{mL}$ of $L$. monocytogenes and used as controls. During the incubation, $0.1 \mathrm{~mL}$ of each culture was taken at $1 \mathrm{~h}$ intervals, diluted as indicated, and then plated on selective medium for L. monocytogenes. At the same time, a light microscope (Olympus-CX31, Olympus, Tokyo, Japan) was used to roughly observe the change in the number of $L$. monocytogenes cells in each sample (Additional file 1: Fig. S1). The number of viable L. monocytogenes cells was counted after $24-48 \mathrm{~h}$ incubation on selective medium at $37{ }^{\circ} \mathrm{C}$. The log reduction was calculated using the following formula: $\log$ reduction $=\log 10$ (number of viable cells before treatment/number of viable cells after treatment).

\section{Scanning electron microscopy (SEM) analysis}

For SEM analysis, spores were purified using a Renografin gradient method to exclude vegetative cells of $B$. subtilis. B. subtilis 168 harboring pUB19-cotG-p75 was cultured for $120 \mathrm{~h}$ and harvested by centrifugation at
$5000 \times g$ for $10 \mathrm{~min}$. The collected pellet was resuspended in $20 \%$ sodium diatrizoate, and then the suspension was layered over $50 \%$ sodium diatrizoate. After centrifugation at $10,000 \times g$ for $30 \mathrm{~min}$, the purified spores were collected at the bottom of the tube. The spores were washed with PBS at least three times to remove any residual sodium diatrizoate.

Sample preparation for SEM was carried out as described by Kockro et al. (2000) with slight modifications. Broth cultures of L. monocytogenes ATCC 19115 $\left(10^{8} \mathrm{CFU} / \mathrm{mL}\right)$ were washed three times with PBS. An aliquot of L. monocytogenes $(100 \mu \mathrm{L})$ was treated with 100 $\mu \mathrm{L}$ of CotG-p75 $\left(10^{8} \mathrm{CFU} / \mathrm{mL}\right)$. After incubation at $37^{\circ} \mathrm{C}$ for $4 \mathrm{~h}$, the specimen was fixed in $2.5 \%(\mathrm{~V} / \mathrm{V})$ glutaraldehyde for $2 \mathrm{~h}$ at $4{ }^{\circ} \mathrm{C}$. The fixed cells were dehydrated through a gradient of $35,50,75,95$, and $100 \%$ ethanol for 10 min each. The dehydrated cells were dried using hexamethyldisilazane (HMDS). Then, the dried cells were coated with gold using a sputter coater (108 auto sputter coater, Cressington Scientific Instruments, Watford, UK). The samples were then observed under a scanning electron microscope (SEM, JSM-7800F Prime from JEOL Ltd., Tokyo, Japan).

\section{Statistical analysis}

All data presented in this study are expressed as mean \pm standard deviation from triplicate samples. The statistical significance of differences between groups was determined by an unpaired two-tailed $t$-test using GraphPad Prism 5 (GraphPad Software Inc., La Jolla, CA, USA) at a significance level of $p<0.05$.

\section{Results}

\section{Construction of the recombinant plasmid}

The recombinant pUB19 plasmid containing the $\cot G$ and $p 75$ genes was constructed and verified by restriction enzyme analysis and PCR (Additional file 1: Fig. S2). The recombinant plasmid was named pUB19-cotG- $p 75$ and a diagram of its structure is shown in Fig. 1. The spores displaying the fusion protein obtained from $B$. subtilis harboring pUB19-cotG-p75 were designated as CotG-p75.

\section{Peptidoglycan hydrolase activity of CotG-p75}

A ninhydrin test was performed to verify that the CotG-p75 displayed on the spore surface maintained its intrinsic PGH activity. PG was treated with different concentrations $\left(1.2,1.8,2.4,3.0\right.$, and $3.6 \times 10^{5} \mathrm{CFU} /$ $\mathrm{mL}$ ) of CotG-p75 and the degree of PG degradation was measured by the ninhydrin test. As shown in Fig. 2, the absorbance increased linearly as the concentration of CotG-p75 increased $\left(R^{2}=0.99\right)$. The PGH activity of the p75 protein fused to the CotG protein seems to be well maintained. Thus, the fusion of the p75 protein with 


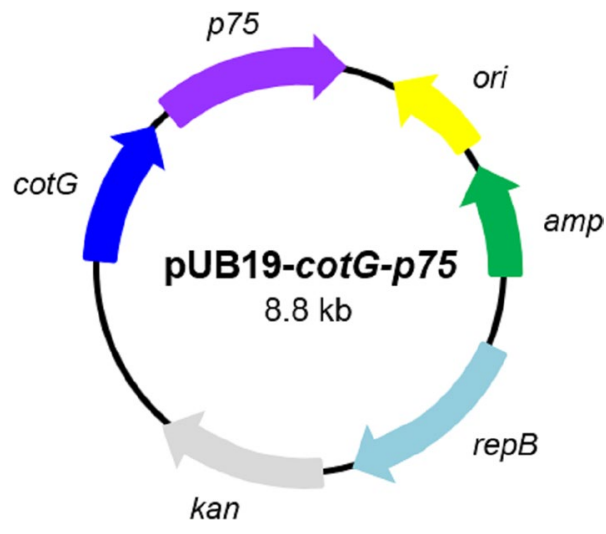

Fig. 1 Plasmid map of the recombinant plasmid pUB19-cotG-p75 The ori and repB represent the replication origin and replication protein $\mathrm{B}$, respectively. The amp and kan represent ampicillin and kanamycin resistance markers, respectively. The $\cot G$ and $p 75$ represent the spore coat protein CotG encoding gene of $B$. subtilis and the 75 protein encoding gene of $L$. rhamnosus $\mathrm{GG}$, respectively

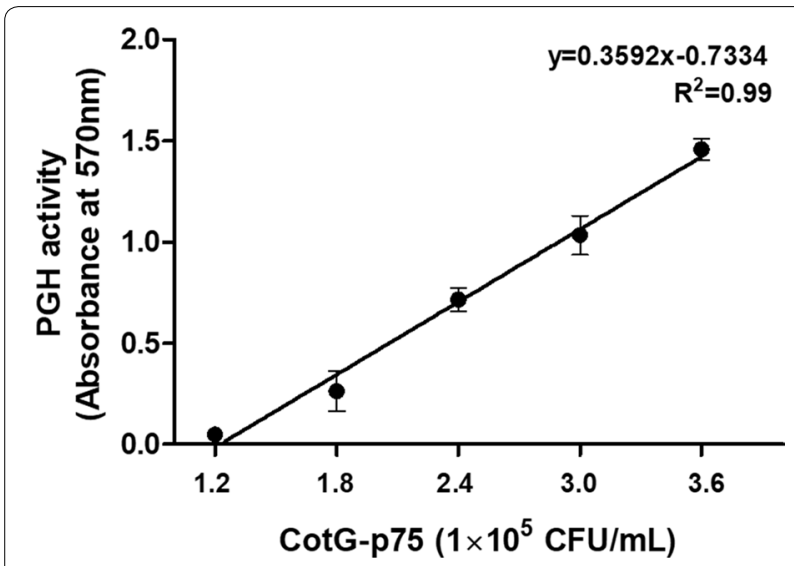

Fig. 2 Determination of the peptidoglycan hydrolase (PGH) activity of CotG-p75 measured by the ninhydrin method. After treatment of peptidoglycan with different concentrations of CotG-p75 at $37^{\circ} \mathrm{C}$ for $15 \mathrm{~min}$, the absorbance of each sample was measured at $570 \mathrm{~nm}$. All tests were performed in triplicate, and the data are presented as mean \pm standard deviation

the CotG protein located on the spore surface does not appear to affect the biological activity of the $\mathrm{p} 75$ protein.

\section{Thermal and pH stability of the spore displayed CotG-p75}

To investigate the stability of CotG-p75, its PGH activity was determined under various temperature and $\mathrm{pH}$ conditions. The PGH activity of CotG-p75 measured at room temperature $\left(25^{\circ} \mathrm{C}\right)$ and $\mathrm{pH} 7$ was used as a control. As shown in Fig. 3a, the PGH activity of the CotG-p75 decreased gradually as the temperature increased from $40{ }^{\circ} \mathrm{C}$ to $80{ }^{\circ} \mathrm{C}$. The activity of CotG-p75 was maintained at more than $90 \%$ at $40{ }^{\circ} \mathrm{C}$ and $70 \%$ and $56 \%$ at $50{ }^{\circ} \mathrm{C}$ and $60{ }^{\circ} \mathrm{C}$, respectively. The CotG-p75 appears to be relatively thermostable and could maintain activity above $40 \%$ even after heat treatment at $80^{\circ} \mathrm{C}$.

The $\mathrm{pH}$ stability of CotG-p75 was also tested after treatment at various $\mathrm{pHs}(2,3,4,5,6,7$, and 8) for $15 \mathrm{~min}$. As shown in Fig. 3b, the activity of CotG-p75 remained stable in the range of $\mathrm{pH} 3-7(p>0.05)$. However, the activity of CotG-p75 was significantly reduced to $6 \%$ at pH 2 , a strong acidic condition, and to $46 \%$ at pH 8 , a relatively weak alkaline condition.

\section{Antibacterial effect of CotG-p75 against L. monocytogenes}

To evaluate the antibacterial effect of CotG-p75, a timekill assay was conducted against $L$. monocytogenes. The CotG-p75 spores at concentrations of $10^{7}, 10^{8}$, and $10^{9} \mathrm{CFU} / \mathrm{mL}$ were mixed with a constant number of $L$. monocytogenes cells $\left(10^{8} \mathrm{CFU} / \mathrm{mL}\right)$ and the viability of the cells was analyzed (Fig. 4). After $6 \mathrm{~h}$ in the control group with PBS or wild-type spores, the viable number of $L$. monocytogenes cells decreased by $0.24 \mathrm{log}$ and 0.44 $\log$, respectively, from the initial cell count. When the ratio of CotG-p75 to L. monocytogenes was 0.1 , the viable cell count of L. monocytogenes decreased by $0.36 \mathrm{log}$, which was not different from the control groups. However, when the CotG-p75/L. monocytogenes ratios were 1 and 10 , the viable cell counts of $L$. monocytogenes tended to decrease rapidly over time and decreased by 1.55 and 2.02 logs after $6 \mathrm{~h}$, respectively. Although the number of p75 proteins displayed on each of the spore's surface was not measured in this study, the viable count of L. monocytogenes clearly decreased as the amount of CotG-p75 increased. The decrease in viable cell number measured by the time-kill assay was roughly confirmed by light microscopy and is presented in Additional file 1: Fig. S1.

\section{Scanning electron microscopy image of $L$. monocytogenes treated with CotG-p75}

In order to confirm whether the antibacterial effect of CotG-p75 was caused by PG degradation, the morphological changes of $L$. monocytogenes treated with CotG-p75 were observed using SEM (Fig. 5). When $L$. monocytogenes cells were exposed to PBS (Fig. 5a) and wild-type spores (Fig. 5b), the surface of L. monocytogenes did not show any significant changes. However, as shown in Fig. 5c, L. monocytogenes cells treated with CotG-p75 for $6 \mathrm{~h}$ exhibited a dented and deformed surface as compared with the controls. In Fig. $5 \mathrm{c}$, it is not clear whether the damaged cells are alive or dead. However, combining this result with the time-kill assay and light microscopy results, the p75 protein displayed on the spore surface appears to have antibacterial activity 

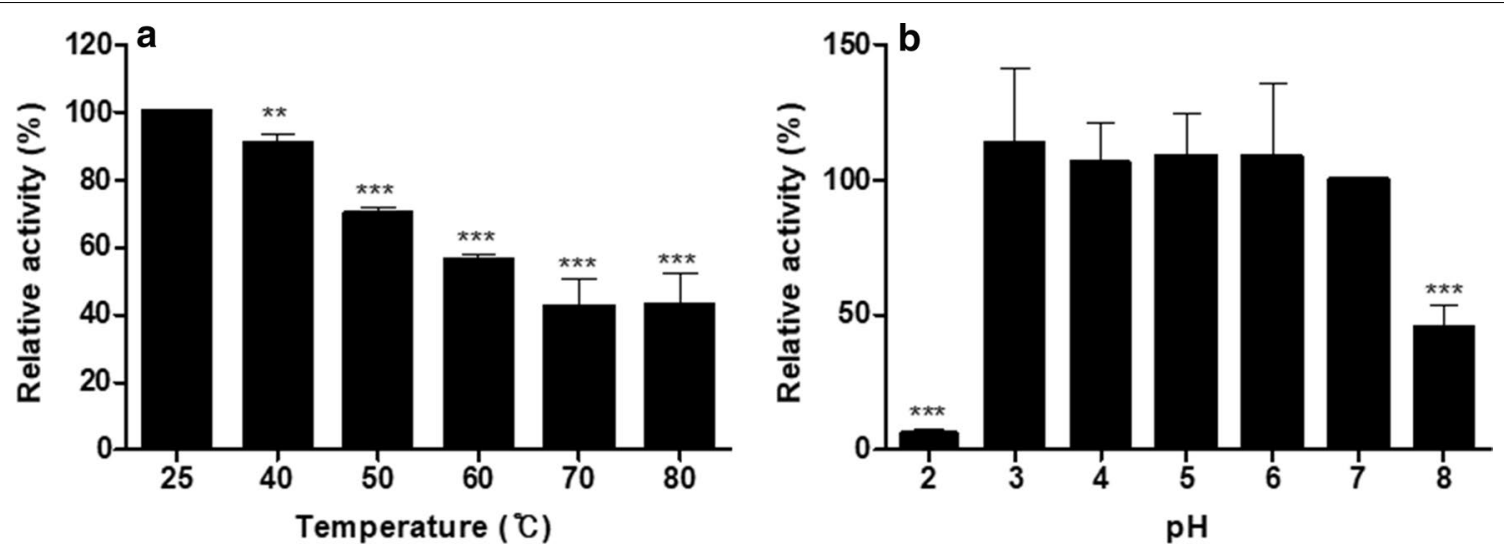

Fig. 3 Relative peptidoglycan hydrolase activity of CotG-p75 after heat (a) and pH (b) treatments. Relative activity was calculated by defining its activity at $25^{\circ} \mathrm{C}$ and $\mathrm{pH} 7$ as 100\%. All tests were performed in triplicate, and the data are presented as mean \pm standard deviation. Statistical analysis was performed by an unpaired two-tailed $t$-test. Asterisks indicate a significance difference from the control $\left({ }^{*} p<0.05,{ }^{* *} p<0.01\right.$, $\left.{ }^{* * *} p<0.001\right)$

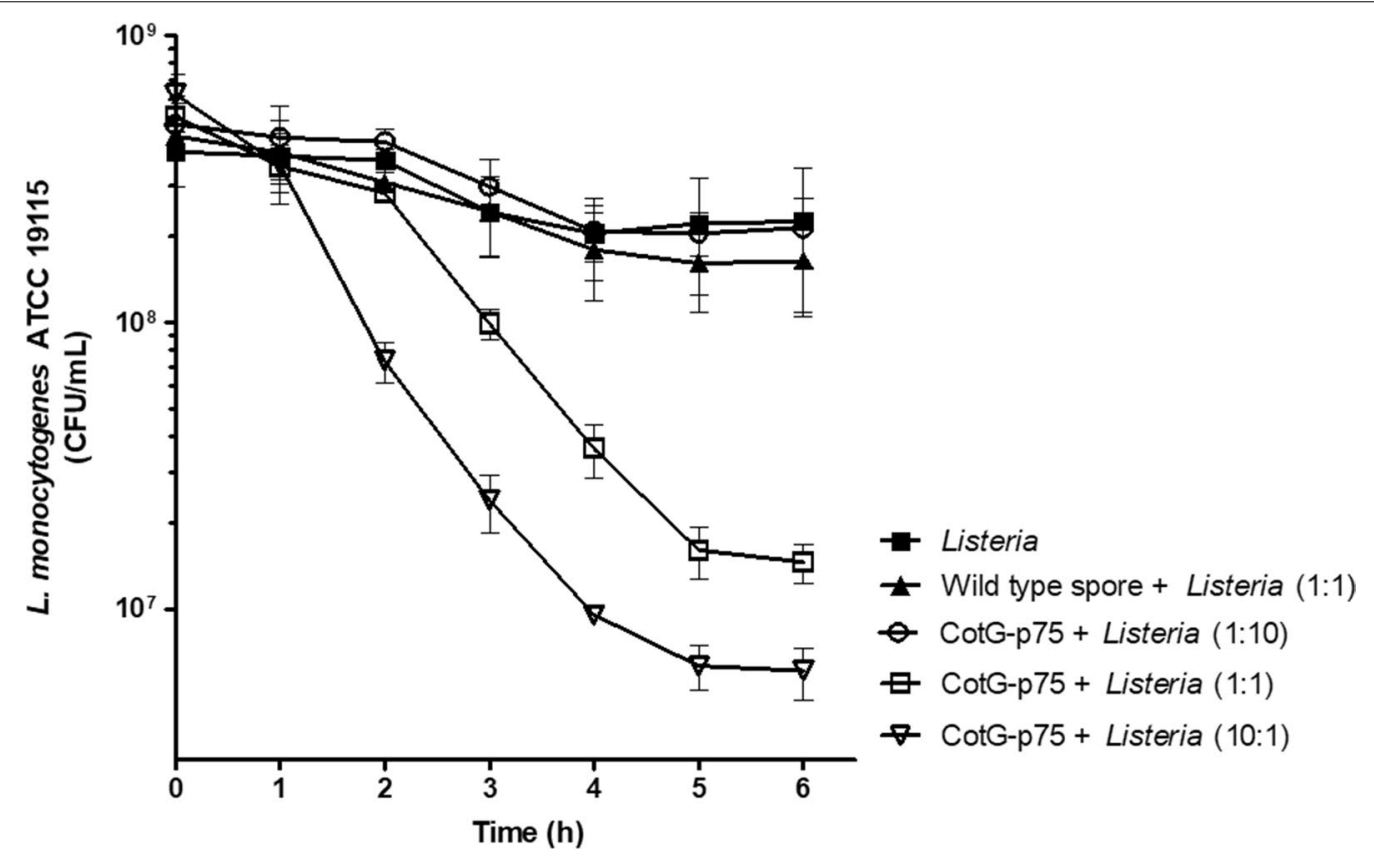

Fig. 4 Antibacterial effect of CotG-p75 against L. monocytogenes. Cells were treated with different concentrations of CotG-p75 in PBS at $37^{\circ} \mathrm{C}$. The control groups were treated with no spores (closed square) or wild-type spores (closed triangle). The number of viable cells was measured at intervals of $1 \mathrm{~h}$ during the $6 \mathrm{~h}$ incubation. All tests were performed in triplicate, and the data are presented as mean \pm standard deviation. Statistical analysis was performed by an unpaired two-tailed t-test

mediated by its effects on the integrity of the cell wall of L. monocytogenes.

\section{Discussion}

Many concerns have been raised about the risks of probiotics such as bacterial infections and sepsis. These risks are not a problem for healthy people but can be fatal for infants, the elderly, and immunocompromised patients
(Boyle et al. 2006). To minimize the risks of bacterial probiotics, proteins secreted by probiotic strains have been proposed as safer and more effective alternatives (Sánchez et al. 2010).

Among the many secreted proteins, p75 is one of the well-known soluble proteins produced by LGG and its probiotic actions have been widely studied. In particular, isolated p75 was more effective than whole bacteria (live 


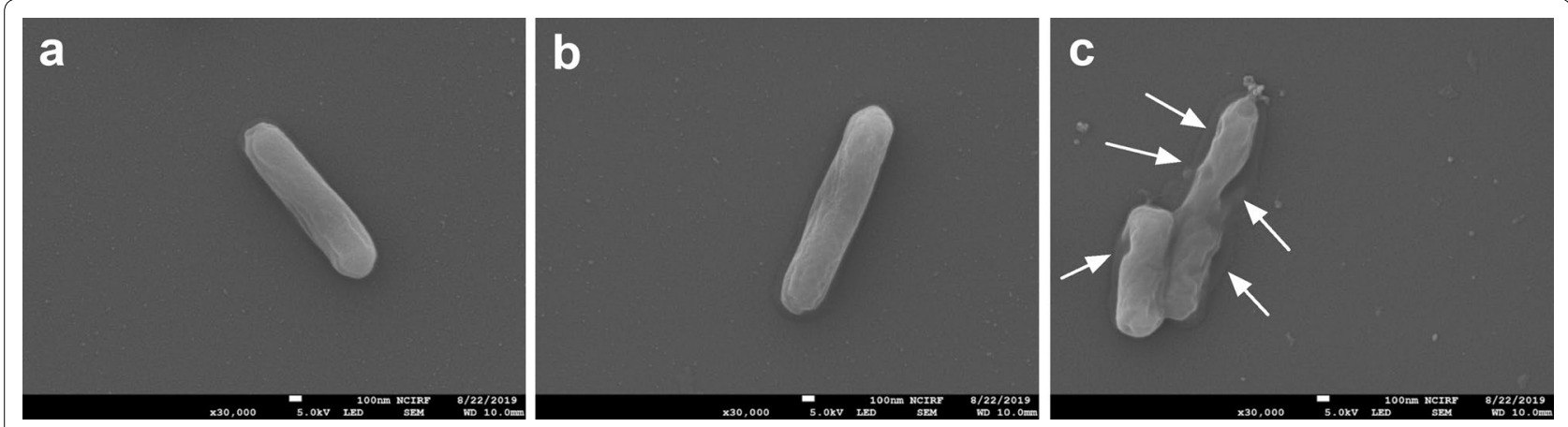

Fig. 5 SEM images of L. monocytogenes untreated (a) and treated with wild type spores (b), and CotG-p75 (c) in PBS for 6 h at $37^{\circ} \mathrm{C}$. Arrows indicate damaged cell surfaces

or heat-killed LGG) in maintaining the homeostasis of intestinal epithelial cells (Seth et al. 2008). However, there are practical limitations to using the purified proteins as a probiotics alternative due to cost and stability issues. In that regard, we chose B. subtilis spores as the vehicle for the probiotic protein p75 to develop new probiotics with improved safety and stability.

Recently, spore surface display technology has been extensively used in the food industries due to its robust resistance in a harsh environment (Zhang et al. 2019). Probiotics need to be highly resistant to various stress conditions, such as manufacturing processes, distribution, storage, and the gastrointestinal transit after ingestion. Therefore, the application of spore surface display technology to probiotics development is expected to improve the stability of probiotics even under harsh conditions.

In this study, we successfully displayed p75 on the surface of B. subtilis spores and verified its stable expression by measuring $\mathrm{PGH}$ activity at a wide range of temperatures and $\mathrm{pH}$. Our results suggested that CotGp75 is a probiotics candidate with stability under a broad spectrum of thermal and $\mathrm{pH}$ conditions. In addition, CotG-p75 showed antibacterial activity against L. monocytogenes but had no effect against Staphylococcus aureus (Additional file 1: Fig. S3).

p75 is known to have homology with PGH, which catalyzes the cleavage of the sugar or amino acid chain of PG (Bäuerl et al. 2010; Claes et al. 2012). PGHs form a very diverse group of enzymes and are involved in the growth and division of bacteria, such as cell wall expansion, PG turnover, and daughter cell separation (Vollmer et al. 2008). Since PGH has bacteriolytic activity, it has been studied as a potential antimicrobial agent to replace conventional antibiotics for prevention of bacterial infection and the development of multi-drug resistance (Parisien et al. 2008). For example, the lysostaphin of Staphylococcus simulans has been extensively studied and is noted for its cell wall lytic effect against Staphylococcus aureus (Baba and Schneewind 1996). Zoocin A of Streptococcus zooepidemicus 4881 and millericin B of Streptococcus milleri NMSCC061 exhibited antibacterial activity against the $S$. aureus group (Simmonds et al. 1996) and a wide range of Gram-positive bacteria, respectively (Beukes et al. 2000). Among the probiotics, the recombinant cell wall hydrolase from Lactobacillus paracasei NRL B-50314 produced by Bacillus megaterium showed an antibacterial effect against Lactobacillus lactis LM 0230 (Liu et al. 2015). The PGH of probiotic Pediococcus acidilactici NCDC 252 also showed cell wall lytic activity against Micrococcus lysodeikticus and Bacillus cereus (Gandhi et al. 2020).

Although many studies have reported the antibacterial effects of PGH on Gram-positive bacteria, not all PGHs have antibacterial activity. The antimicrobial activity of bacterial PGH has a substrate specificity depending on the enzyme source and it exhibits a narrow spectrum that functions against only certain bacterial species or subspecies (Parisien et al. 2008).

The p75 proteins of $L$. casei and L. rhamnosus are known to belong to a large family of secreted cell wall proteins with PGH activity (Bäuerl et al. 2019). The purified p75 protein from $L$. casei has no significant lytic effect against Enterococcus faecalis, L. monocytogenes, or S. aureus (Bäuerl et al. 2019). This result is somewhat different from the results of this study, in which CotG-p75 showed antibacterial effects against $L$. monocytogenes. In L. casei and L. rhamnosus, the C-terminal amino acid sequence of the p75 protein is well conserved between the two species, but the sequences of the N-terminus region are quite different (Bäuerl et al. 2010). In the previous studies, truncation of the cell wall binding domain, which is usually located in the $\mathrm{N}$-terminus of $\mathrm{PGH}$, reduced its autolytic activity 
(Gargis et al. 2010; Osipovitch and Griswold 2015). Therefore, the observed differences in the antibacterial activity of $\mathrm{p} 75$ protein against $L$. monocytogenes may be due to the differences between the PGHs produced by the two species, L. casei and L. rhamnosus. In addition, in the case of CotG-p75, the N-terminus of the p75 was directly connected to the C-terminus of the CotG protein, and thus the possibility that the structure of the p75 was affected cannot be excluded.

In conclusion, the p75 protein of L. rhamnosus GG was successfully displayed on $B$. subtilis spores with CotG as an anchoring motif. Stable expression of CotGp75 was demonstrated with PGH activity in a wide range of temperature and $\mathrm{pH}$ conditions. The CotG-p75 showed antibacterial activity against $L$. monocytogenes, probably due to the cell wall hydrolytic activity of $\mathrm{p} 75$. The results suggest that the spore surface-displayed CotG-p75 may be a potential antimicrobial agent against L. monocytogenes. However, developing CotGp75 as a new probiotics candidate requires additional research and evaluation of its probiotic activity.

\section{Supplementary information}

Supplementary information accompanies this paper at https://doi. org/10.1186/s13568-020-01073-9.

Additional file 1. Additional figures.

\section{Abbreviations}

ATCC: American type culture collection; CFU: Colony forming units; LGG: Lactobacillus rhamnosus GG; PBS: Phosphate-buffered saline; PCR: Polymerase chain reaction; PG: Peptidoglycan; PGH: Peptidoglycan hydrolase; SDS: Sodium dodecyl sulfate; SEM: Scanning electron microscopy.

\section{Acknowledgements}

Not applicable.

\section{Authors' contributions}

SJK, JSJ, and JAM carried out the experiment. SJK wrote the manuscript with support from JSJ and JAM. KWH supervised this work. All authors read and approved the final manuscript.

\section{Funding}

This work was supported under the framework of international cooperation program managed by National Research Foundation of Korea (NRF-2018R1D1A1A02086040).

\section{Availability of data and materials}

The datasets supporting the conclusions of this article are included within the article and its additional files.

\section{Ethics approval and consent to participate}

This article does not contain any studies with human participants or animals performed by any of the authors.

\section{Consent for publication}

Not applicable.

\section{Competing interests}

The authors declare that they have no competing interests.
Received: 10 April 2020 Accepted: 25 July 2020

Published online: 08 August 2020

\section{References}

Anagnostopoulos C, Crawford IP (1961) Transformation studies on the linkage of markers in the tryptophan pathway in Bacillus subtilis. PNAS 47:378-390

Atrih A, Bacher G, Allmaier G, Williamson MP, Foster SJ (1999) Analysis of peptidoglycan structure from vegetative cells of Bacillus subtilis 168 and role of PBP 5 in peptidoglycan maturation. J Bacteriol 181(3):3956-3966

Baba T, Schneewind O (1996) Target cell specificity of a bacteriocin molecule: a C-terminal signal directs lysostaphin to the cell wall of Staphylococcus aureus. EMBO J 15(18):4789

Barry AL, Craig WC, Nadler H, Reller LB, Sanders CC, Swenson JM (1999) Methods for determining bactericidal activity of antimicrobial agents; approved guideline. NCCLS document M26-A. National Committee for Clinical Laboratory Standards, Wayne, Pennsylvania

Bäuerl C, Perez-Martinez G, Yan F, Polk DB, Monedero V (2010) Functional analysis of the p40 and p75 proteins from Lactobacillus casei BL23. J Mol Microb Biotech 19:231-241

Bäuerl C, Abitayeva G, Sosa-Carrillo S, Mencher-Beltrán A, Navarro-Lleó N, CollMarqués JM, Zúñiga-Cabrera M, Shaikhin S, Pérez-Martinez G (2019) P40 and P75 are singular functional muramidases present in the Lactobacillus casei/paracasei/rhamnosus taxon. Front Microbiol 10:1420

Beukes M, Bierbaum G, Sahl HG, Hastings J (2000) Purification and partial characterization of a murein hydrolase, millericin B, produced by Streptococcus milleri NMSCC 061. Appl Environ Microb 66(1):23-28

Bomba A, Nemcová R, Mudronová D, Guba P (2002) The possibilities of potentiating the efficacy of probiotics. Trends Food Sci Tech 13:121-126

Boyle RJ, Robins-Browne RM, Tang ML (2006) Probiotic use in clinical practice: what are the risks? Am J Clin Nutr 83(6):1256-1447

Chen HL, Lai YW, Chen CS, Chu TW, Lin W, Yen CC, Lin MF, Tu MY, Chen CM (2010) Probiotic Lactobacillus casei expressing human lactoferrin elevates antibacterial activity in the gastrointestinal tract. Biometals 23:543-554

Chen H, Ullah J, Jia J (2017) Progress in Bacillus subtilis spore surface display technology towards environment, vaccine development, and biocatalysis. J Mol Microb Biotech 27(3):159-167

Claes IJ, Schoofs G, Regulski K, Courtin P, Chapot-Chartier MP, Rolain T, Hols P, von Ossowski I, Reunanen J, de Vos WM, Palva A, Vanderleyden J, De Keersmaecker SC, Lebeer S (2012) Genetic and biochemical characterization of the cell wall hydrolase activity of the major secreted protein of Lactobacillus rhamnosus GG. PLoS ONE 7:31588

Cutting SM (2011) Bacillus probiotics. Food Microbiol 28:214-220

Doron S, Snydman DR, Gorbach SL (2005) Lactobacillus GG: bacteriology and clinical applications. Gastroenterol Clin N 34:483-498

Elshaghabee FMF, Rokana N, Gulhane RD, Sharma C, Panwar H (2017) Bacillus as potential probiotics: status, concerns, and future perspectives. Front Microbiol 8:1490

Gandhi D, Chanalia P, Bansal P, Dhanda S (2020) Peptidoglycan hydrolases of probiotic Pediococcus acidilactici NCDC 252: isolation, physicochemical and in silico characterization. IntJ Pept Res Ther. https://doi.org/10.1007/ s10989-019-10008-3

Gargis SR, Heath HE, LeBlanc PA, Dekker L, Simmonds RS, Sloan GL (2010) Inhibition of the activity of both domains of lysostaphin through peptidoglycan modification by the lysostaphin immunity protein. Appl Environ Microbiol 76(20):6944-6946

Goh YL, He H, March JC (2012) Engineering commensal bacteria for prophylaxis against infection. Curr Opin Biotechn 23:924-930

Guo Q, An Y, Yun J, Yang M, Magocha TA, Zhu J, Xue Y, Qi Y, Hossain Z, Sun W, Qi X (2018) Enhanced d-tagatose production by spore surface displayed L-arabinose isomerase from isolated Lactobacillus brevis PC16 and biotransformation. Bioresour Technol 247:940-946

Hanahan D (1983) Studies on transformation of Escherichia coli with plasmids. J Mol Biol 166(4):557-580

He W, Jiang B, Mu W, Zhang T (2016) Production of d-allulose with d-psicose 3-epimerase expressed and displayed on the surface of Bacillus subtilis spores. J Agric Food Chem 64:7201-7207

Henriques AO, Moran CP Jr (2007) Structure, assembly, and function of the spore surface layers. Annu Rev Microbiol 61:555-588 
Hong HA, Duc LH, Cutting SM (2005) The use of bacterial spore formers as probiotics. FEMS Microbiol Rev 29:813-835

Juhas M, Ajioka JW (2016) Integrative bacterial artificial chromosomes for DNA integration into the Bacillus subtilis chromosome. J Microbiol Methods 125:1-7

Kang SJ, Park EA, Lee DH, Hong KW (2019) Comparison of the stability of eGFP displayed on the Bacillus subtilis spore surface using CotB and C-terminally truncated CotB proteins as an anchoring motif under extreme conditions. Appl Biol Chem 62:41

Karimi O, Peña AS (2008) Indications and challenges of probiotics, prebiotics, and synbiotics in the management of arthralgias and spondyloarthropathies in inflammatory bowel disease. J Clin Gastroenterol 42:136-141

Kim J, Schumann W (2009) Display of proteins on Bacillus subtilis endospores. Cell Mol Life Sci 66:3127-3136

Kockro RA, Hampl JA, Jansen B, Peters G, Scheihing M, Giacomelli R, Kunze S, Aschoff A (2000) Use of scanning electron microscophy to investigate the prophylactic efficacy of rifampin-impregnated CSF shunt catheters. J Med Microbiol 49:441-450

Liu Y, Li S, Xu H, Wu L, Xu Z, Liu J, Feng X (2014) Efficient production of D-tagatose using a food-grade surface display system. J Agric Food Chem 62:6756-6762

Liu S, Rich JO, Anderson A (2015) Antibacterial activity of a cell wall hydrolase from Lactobacillus paracasei NRRL B-50314 produced by recombinant Bacillus megaterium. J Ind Microbiol Biot 42:229-235

Liu H, Yang S, Wang X, Wang T (2019) Production of trehalose with trehalose synthase expressed and displayed on the surface of Bacillus subtilis spores. Microb Cell Fact 18(1):100

Mattila-Sandholm T, Myllärinen P, Crittenden R, Mogensen G, Fondén R, Saarela M (2002) Technological challenges for future probiotic foods. Int Dairy J 12(2-3):173-182

Moore S, Stein WH (1948) Photometric ninhydrin method for use in the chromatography of amino acids. J Biol Chem 176(1):367-388

Morrow LE, Kollef MH (2008) Probiotics in the intensive care unit: why controversies and confusion abound. Crit Care 12:160

Nicholson WL, Setlow P (1990) Dramatic increase in negative superhelicity of plasmid DNA in the forespore compartment of sporulating cells of Bacillus subtilis. J Bacteriol 172(1):7-14

Nicholson WL, Munakata N, Horneck G, Melosh HJ, Setlow P (2000) Resistance of Bacillus endospores to extreme terrestrial and extraterrestrial environments. Microbiol Mol Biol R 64(3):548-572

Osipovitch D, Griswold K (2015) Fusion with a cell wall binding domain renders autolysin LytM a potent anti-Staphylococcus aureus agent. FEMS Microbiol Lett 362(2):1-7

Parisien A, Allain B, Zhang J, Mandeville R, Lan CQ (2008) Novel alternatives to antibiotics: bacteriophages, bacterial cell wall hydrolases, and antimicrobial peptides. J Appl Microbiol 104(1):1-13

Sambrook J, Fritsch ED, Maniatis T (1989) Molecular cloning: A laboratory manual, 2nd edn. Cold Spring Harbor Laboratory Press, New York, Cold Spring Harbor, pp 49-55
Sánchez B, Urdaci MC, Margolles A (2010) Extracellular proteins secreted by probiotic bacteria as mediators of effects that promote mucosa-bacteria interactions. Microbiology 156(11):3232-3242

Seth A, Yan F, Polk DB, Rao RK (2008) Probiotics ameliorate the hydrogen peroxide-induced epithelial barrier disruption by a PKC-and MAP kinasedependent mechanism. Am J Physiol-Gastr 294:1060-1069

Sheehan VM, Sleator RD, Fitzgerald GF, Hill C (2006) Heterologous expression of BetL, a betaine uptake system, enhances the stress tolerance of Lactobacillus salivarius UCC118. Appl Environ Microbiol 72:2170-2177

Sheehan VM, Sleator RD, Hill C, Fitzgerald GF (2007) Improving gastric transit, gastrointestinal persistence and therapeutic efficacy of the probiotic strain Bifidobacterium breve UCC2003. Microbiology 153:3563-3571

Simmonds R, Pearson L, Kennedy R, Tagg J (1996) Mode of action of a lysostaphin-like bacteriolytic agent produced by Streptococcus zooepidemicus 4881. Appl Environ Microbiol 62(12):4536-4541

Szajewska H, Wanke M, Patro B (2011) Meta-analysis: the effects of Lactobacillus rhamnosus GG supplementation for the prevention of healthcareassociated diarrhoea in children. Aliment Pharmacol Ther 34:1079-1087

Termont S, Vandenbroucke K, Iserentant D, Neirynck S, Steidler L, Remaut E, Rottiers P (2006) Intracellular accumulation of trehalose protects Lactococcus lactis from freeze-drying damage and bile toxicity and increases gastric acid resistance. Appl Environ Microbiol 72(12):7694-7700

Vollmer W, Blanot D, de Pedro MA (2008) Peptidoglycan structure and architecture. FEMS Microbiol Rev 32:149-167

Wang H, Yang R, Hua X, Zhang W, Zhao W (2016) Production using the CotXmediated spore-displayed beta-galactosidase as a biocatalyst. J Microbiol Biotechn 26:1267-1277

Wriggers W, Chakravarty S, Jennings PA (2005) Control of protein functional dynamics by peptide linkers. Biopolymers 80:736-746

Xu X, Gao C, Zhang X, Che B, Ma C, Qiu J, Tao F, Xu P (2011) Production of $\mathrm{N}$-acetyl-D-neuraminic acid by use of an efficient spore surface display system. Appl Environ Microbiol 77:3197-3201

Yan F, Polk DB (2002) Probiotic bacterium prevents cytokine-induced apoptosis in intestinal epithelial cells. J Biol Chem 277:50959-50965

Yan F, Cao H, Cover TL, Whitehead R, Washington MK, Polk DB (2007) Soluble proteins produced by probiotic bacteria regulate intestinal epithelial cell survival and growth. Gastroenterology 132:562-575

Zhang G, An Y, Zabed HM, Guo Q, Yang M, Yuan J, Li W, Sun W, Qi X (2019) Bacillus subtilis spore surface display technology: a review of its development and applications. J Microbiol Biotechn 29(2):179-190

Zocco MA, dal Verme LZ, Cremonini F, Piscaglia AC, Nista EC, Candelli M, Novi M, Rigante D, Cazzato IA, Ojetti V, Armuzzi A, Gasbarrini G, Gasbarrini A (2006) Efficacy of Lactobacillus GG in maintaining remission of ulcerative colitis. Aliment Pharmacol Ther 23:1567-1574

\section{Publisher's Note}

Springer Nature remains neutral with regard to jurisdictional claims in published maps and institutional affiliations.

\section{Submit your manuscript to a SpringerOpen ${ }^{\circ}$ journal and benefit from:}

- Convenient online submission

- Rigorous peer review

- Open access: articles freely available online

- High visibility within the field

Retaining the copyright to your article

Submit your next manuscript at springeropen.com 